August 1997

WATPHYS-TH-97/13

hep-th/9709064

\title{
Universality of Quantum Entropy for Extreme Black Holes
}

\author{
Robert B. Mann \\ Department of Physics, University of Waterloo, Waterloo, Ontario N2L 3G1, Canada
}

\begin{abstract}
We consider the extremal limit of a black hole geometry of the Reissner-Nordstrom type and compute the quantum corrections to its entropy. Universally, the limiting geometry is the direct product of two 2-dimensional spaces and is characterized by just a few parameters. We argue that the quantum corrections to the entropy of such extremal black holes due to a massless scalar field have a universal behavior. We obtain explicitly the form of the quantum entropy in this extremal limit as function of the parameters of the limiting geometry. We generalize these results to black holes with toroidal or higher genus horizon topologies. In general, the extreme quantum entropy is completely determined by the spectral geometry of the horizon and in the ultra-extreme case it is just a determinant of the 2-dimensional Laplacian. As a byproduct of our considerations we obtain expressions for the quantum entropy of black holes which are not of the Reissner-Nordstrom type: the extreme dilaton and extreme Kerr-Newman black holes. In both cases the classical Bekenstein-Hawking entropy is modified by logarithmic corrections.
\end{abstract}

*e-mail: mann@avatar.uwaterloo.ca

${ }^{\dagger}$ e-mail: sergey@avatar.uwaterloo.ca 
The origin of black hole entropy remains an exciting unresolved problem requiring implementation of new ideas and deep insights. Of particular interest is the physics of extremal black holes and their entropy. Taking the extremal limit directly in the Bekenstein-Hawking (BH) expression [1] on finds that entropy of an extreme black hole is proportional to the area of its horizon

$$
S_{B H}^{e x t}=\frac{A}{4 G}
$$

as it is in the non-extreme case. The same situation holds for the quantum corrections to the extremal BH entropy. In particular, quantum corrections due to a minimal scalar field yield UV-divergent contributions to the entropy of a Reissner-Nordstrem (RN) black hole which in the extreme limit take the form [2]

$$
S_{q}^{e x t}=\frac{A}{48 \pi \epsilon^{2}}+\frac{1}{90} \ln \left(\frac{\epsilon}{\Lambda}\right),
$$

where $\epsilon$ is the ultraviolet (UV) cut-off and $\Lambda$ is some length scale associated with the black hole metric.

However, it was proposed recently [3] that extreme black holes actually must have zero entropy, $S^{e x t}=0$, and can be in thermal equilibrium at an arbitrary temperature. The reason for this is purely topological. In the extreme case the Euclidean space-time near the horizon has the form of an infinite throat that allows one to identify the Euclidean time variable with an arbitrary period $T^{-1}$, thereby implying that its Lorentzian continuation corresponds to an extremal black hole with an arbitrary temperature $T$. This differs from the non-extreme case where for Euclidean time with an arbitrary period $T^{-1}$, a conical singularity at the horizon is present in the Euclidean section, disappearing only for the Hawking temperature $T=T_{H}$.

However subsequent study of extremal black holes in superstring theory [i] indicates that their entropy can be understood to arise from degrees of freedom that correspond to basic string states. This gives strong support to the first viewpoint, namely that the entropy of an extreme black hole is proportional to its horizon area. Whether or not this viewpoint is consistent with the topological arguments of ref. [3] remains unclear.

Recently, Zaslavskii [5 proposed a method for reaching the extremal limit of a black holes whilst remaining in the topological class of the corresponding non-extremal geome- 
tries. He considered a sequence of non-extreme black holes in a cavity at $r=r_{B}$ and found that there exists a set of data $\left(r_{+}, r_{B}, r_{-}\right)$such that the limit $\frac{r_{+}}{r_{-}} \rightarrow 1, \frac{r_{B}}{r_{+}} \rightarrow 1$ is well-defined. The resultant limiting geometry belongs to the non-extremal topological class and takes the form of a direct product of two 2-dimensional spaces. Moreover, there is a remarkable universality. No matter how arbitrary the non-extreme geometry is, the limiting extremal one is characterized by just a few parameters: a pair of dimensional ones, $r_{+}$and $\bar{b}$, and one dimensionless parameter $c$. For $c=1$ the limiting geometry is the direct product of 2-dimensional hyperbolic space $\left(H_{2}\right)$ with the two-dimensional sphere $\left(S_{2}\right)$, where $\left(\bar{b}^{-1 / 2}\right.$ and $r_{+}$are the respective radii of $H_{2}$ and $\left.S_{2}\right)$. Since the limiting geometry belongs to the non-extreme class its entropy is necessarily proportional to the horizon area in accord with the $\mathrm{BH}$ formula.

The classical BH entropy (11) is actually a tree-level quantity. In a theory of gravity whose Lagrangian contains terms quadratic in curvature, the entropy is modified by terms which are not proportional to the horizon area and depend upon the external geometry of the horizon [6]. Any quantum field propagating in the black hole background adds some (one-loop) correction $S_{q}$ to the entropy. Generally, this correction is not determined only by the horizon geometry and is a complicated functional of the background metric. Very little is known about it in general, and the exact form of this functional can be retrieved only in some special cases. In particular, it is known in two dimensions [7] for conformal scalar matter and in three dimensions 8 for a scalar field on the BTZ background.

The universality we mentioned above suggests that the quantum entropy $S^{B H}$ of a black hole possesses a universal behavior in its extreme limit going to some limiting function $S^{B H} \rightarrow S_{q}^{e x t}\left(r_{+}, b, c\right)$. The fact that for $c=1$ the limiting geometry has an especially simple form, $H_{2} \times S_{2}$, suggests that in this case the limiting function $S_{q}^{\text {ext }}\left(r_{+}, b\right)$ can be found explicitly.

The purpose of this paper is to demonstrate these results. We start first with a brief review of the limiting procedure of [5] and then calculate the effective action and the quantum entropy due to a minimal scalar field. We use the heat kernel technique which for the present problem proceeds in a manner similar to that developed in ref. [8] for the $(2+1)$-dimensional BTZ black hole. We refer the reader to the ref. 8 for technical 
details.

Before proceeding, we pause to note that an earlier attempt 9] at computing quantum corrections to the entropy of topologically extremal Reissner-Nordstrom black holes [3] found that these were proportional to powers of the temperature and had divergences that were no stronger than in the non-extremal case. The renormalization approach employed implied that the temperature necessarily vanished, suggesting that these quantum corrections to the entropy in the extremal case also vanished. However such a value is not a smooth continuation of the non-extremal case.

Consider an arbitrary static spheri-symmetric geometry

$$
\begin{aligned}
& d s^{2}=g(r) d \tau^{2}+\frac{1}{f(r)} d r^{2}+r^{2} d \omega^{2}, \\
& d \omega^{2}=d \theta^{2}+\sin ^{2} \theta d \varphi^{2}
\end{aligned}
$$

describing a non-extreme hole with an outer horizon located at $r=r_{+}$. Then, the functions $g(r)$ and $f(r)$ in (3) can be expanded as follows

$$
\begin{aligned}
& f(r)=a\left(r-r_{+}\right)+b\left(r-r_{+}\right)^{2}+O\left(\left(r-r_{+}\right)^{2}\right) \\
& g(r)=g_{0}\left(r-r_{+}\right)+g_{1}\left(r-r_{+}\right)^{2}+O\left(\left(r-r_{+}\right)^{2}\right)
\end{aligned}
$$

It is convenient to consider the geodesic distance $l=\int f^{-1 / 2} d r$ as a radial coordinate. Retaining the first two terms in the first of equations (幽), we find for $r>r_{+}$that

$$
\left(r-r_{+}\right)=\frac{a}{b} \sinh ^{2}\left(\frac{l b^{1 / 2}}{2}\right)
$$

and that

$$
g\left(l b^{1 / 2}\right)=\left(\frac{a}{b}\right)^{2} g_{1} \sinh ^{2}\left(\frac{l b^{1 / 2}}{2}\right)\left(c+\sinh ^{2}\left(\frac{l b^{1 / 2}}{2}\right)\right)
$$

where $c=g_{0} b / a g_{1}$ is a dimensionless parameter. In order to avoid appearance of a conical singularity at $r=r_{+}$the Euclidean time $\tau$ in (3) must be identified with period $4 \pi / \sqrt{a g_{0}}$ which goes to infinity in the extreme limit $a \rightarrow 0$. However, rescaling $\tau \rightarrow \phi=\tau \sqrt{a g_{0}} / 2$ yields a new variable $\phi$ having period $2 \pi$. Then, taking into account (5) and (6) we find for the metric (3)

$$
\begin{aligned}
& d s^{2}=\frac{1}{b}\left(g(x) d \phi^{2}+d x^{2}\right)+\left(r_{+}+\frac{a}{b} \sinh ^{2} \frac{x}{2}\right)^{2} d \omega^{2}, \\
& g(x)=4 \sinh ^{2}\left(\frac{x}{2}\right)\left(1+\frac{1}{c} \sinh ^{2} \frac{x}{2}\right),
\end{aligned}
$$


where we have introduced the variable $x=l b^{1 / 2}$.

To obtain the extremal limit we take $a \rightarrow 0$ keeping $c$ finite (i.e. the ratio $a / g_{0}$ must remain finite). The limiting geometry

$$
d s^{2}=\frac{1}{b}\left(g(x) d \phi^{2}+d x^{2}\right)+r_{+}^{2} d \omega^{2}
$$

is that of the direct product of two 2-dimensional spaces and is characterized by a pair of dimensional parameters $b^{-1 / 2}$ and $r_{+}$and one dimensionless parameter $c$. The parameter $r_{+}$sets the length scale for the 2-dimensional space of constant curvature described by the 2 -metric $d \omega^{2}$. The parameter $b^{-1 / 2}$ performs the same job for the $(x, \phi) 2$-space, and the parameter $c$ provides a dimensionless measure of how far this 2-space departs from one of constant negative curvature. This is the universality we mentioned above: although the non-extreme geometry is in general described by an infinite number of parameters associated with the determining functions $f(r)$ and $g(r)$ the geometry in the extreme limit depends only on three parameters $b, r_{+}, c$. Note that the coordinate $r$ is inadequate for describing the extremal limit (8) since the coordinate transformation (5) is singular when $a \rightarrow 0$.

The limiting geometry (8) takes especially simple form if $g(r)=f(r)$. Then $c=1$ and the extreme metric

$$
d s^{2}=\frac{1}{b}\left(\sinh ^{2} x d \phi^{2}+d x^{2}\right)+r_{+}^{2} d \omega^{2}
$$

is that of a direct product $H_{2} \times S_{2}$ of $2 \mathrm{~d}$ hyperbolic space $H_{2}$ with radius $l=b^{-1 / 2}$ and a $2 \mathrm{~d}$ sphere $S_{2}$ with radius $l_{1}=r_{+}$. We shall consider this case below. It is worth noting that the limiting geometry (8) (or (9)) precisely merges near the horizon with the geometry of the original metric (3) in the sense that all the curvature tensors for both metrics coincide. This is in contrast with, say, the situation in which the Rindler metric is considered to approximate to geometry of a non-extreme black hole: the curvatures of both spaces do not merge in general.

For the Reissner-Nordstrom black hole we have $b^{-1}=r_{+}^{2}$ and the limiting extreme geometry is the well-known Bertotti-Robinson space characterized by just one parameter $r_{+}$. This space has remarkable properties in the context of supergravity theory. In particular, the effective action of $N=2$ supergravity was shown [10] to acquire no quantum 
corrections in the background of this metric. These supersymmetry aspects, though intriguing, are not the subject of interest in this paper and we shall not consider them any further.

In the past year there has been an active study of generalizations of the metric (3) which describe black holes with horizons of non-trivial topology [11]. In this generalization the spherical element $d \omega^{2}$ in (3) is replaced by either the flat element

$$
d \omega_{T}^{2}=d \theta^{2}+d \varphi^{2}
$$

or the hyperbolic element

$$
d \omega_{H}^{2}=d \theta^{2}+\sinh ^{2} \theta d \varphi^{2}
$$

describing a space with constant negative curvature, and the coordinates in this space are appropriately identified so as to make the space both compact and free of conical singularities. Remarkably, the metric (3) with such "angle" elements is still a solution of the Einstein equations with a cosmological term. Asymptotically, however, it does not have the topology of flat space. After the appropriate identifications in the $(\theta, \varphi)$ section, the metrics (10) and (11) describe respectively a torus $T=\Sigma_{g=1}$ and a surface $\Sigma_{g}$ of genus $g \geq 2$ respectively [11]. Since the element (10) or (11) describes the metric on the horizon we observe that the horizon may have a non-trivial topology characterized by an arbitrary genus $g$. The limiting procedure described above goes through for these types of black holes and in general we obtain the limiting metric

$$
d s^{2}=\frac{1}{b}\left(g(x) d \phi^{2}+d x^{2}\right)+r_{+}^{2} d \omega_{(g)}^{2}
$$

where $d \omega_{(g)}^{2}$ is the metric on a $2 \mathrm{~d}$ compact surface of genus $g$. The extreme geometry now is $E_{g}=H_{2} \times \Sigma_{g}$. All our formulas may be generalized to include arbitrary $g$.

Note also that not all the known black holes are described by a metric of the form (3). Exceptions include static dilaton black holes and the stationary Kerr-Newman black holes. For these cases the limiting procedure must be reformulated. However, insofar as the entropy calculation is concerned, our computations below provide us with a hint as to how in some cases the total renormalized entropy can be obtained even without knowledge of the limiting geometry. One needs only to know the UV divergences of the quantum entropy. These have been obtained in the earlier studies [2], [16], [17], [9], [18]. 
Consider now a scalar field minimally coupled to gravity. Quantizing it on the black hole background (9) yields quantum corrections to the black hole entropy. It is these corrections that we wish to compute for extremal black holes. We proceed by allowing the coordinate $\phi$ which plays the role of Euclidean time to have period $2 \pi \alpha$. For $\alpha \neq 1$ the metric (9) then describes the space $E^{\alpha}=H_{2}^{\alpha} \times S_{2}$, where $H_{2}^{\alpha}$ is the hyperbolic space coinciding with $H_{2}$ everywhere except the point $x=0$ where it has a conical singularity with an angular deficit $\delta=2 \pi(1-\alpha)$.

The effective action of the scalar field

$$
W_{e f f}=-\frac{1}{2} \int_{\epsilon^{2}}^{\infty} \frac{d s}{s} \operatorname{Tr} K
$$

is expressed by means of the trace of the heat kernel $K\left(z, z^{\prime}, s\right)$ satisfying the heat equation

$$
\begin{aligned}
& \left(\partial_{s}-\square\right) K\left(z, z^{\prime}, s\right)=0, \\
& K\left(z, z^{\prime}, s=0\right)=\delta\left(z, z^{\prime}\right),
\end{aligned}
$$

where $\square=\nabla_{\mu} \nabla^{\mu}$ is the Laplace operator. A nice property of the heat kernel is that it factorizes on a product space. Thus, for the heat kernel on $E^{\alpha}$ we have

$$
K_{E^{\alpha}}\left(z, z^{\prime}, s\right)=K_{H_{2}^{\alpha}}\left(x, x^{\prime}, \phi, \phi^{\prime}, s\right) K_{S^{2}}\left(\theta, \theta^{\prime}, \varphi, \varphi^{\prime}, s\right)
$$

and the effective action reads

$$
W_{e f f}\left[E^{\alpha}\right]=-\frac{1}{2} \int_{\epsilon^{2}}^{\infty} \frac{d s}{s} \operatorname{Tr} K_{H_{2}^{\alpha}} \operatorname{Tr} K_{S_{2}}
$$

where $\epsilon$ is a UV cut-off. On spaces with constant curvature the heat kernel function is known explicitly [12]. In particular, on a $2 \mathrm{~d}$ space with constant negative curvature $R=-\frac{2}{l^{2}}$

$$
K_{H_{2}}\left(z, z^{\prime}, s\right)=\frac{1}{l^{2}} \frac{\sqrt{2} e^{-\bar{s} / 4}}{(4 \pi \bar{s})^{3 / 2}} \int_{\sigma}^{\infty} \frac{d y y e^{-y^{2} / 4 \bar{s}}}{\sqrt{\cosh y-\cosh \sigma}}
$$

where $\bar{s}=s l^{-2}$. The corresponding expression on a $2 \mathrm{~d}$ sphere (which has constant positive curvature $\left.R=\frac{2}{l_{1}^{2}}\right)$ is

$$
K_{S_{2}}\left(z, z^{\prime}, s\right)=\frac{1}{l_{1}^{2}} \frac{\sqrt{2} e^{-\bar{s} / 4}}{(4 \pi \bar{s})^{3 / 2}} \sum_{n=-\infty}^{\infty}(-1)^{n} \int_{\sigma}^{\pi} \frac{d \varphi(\varphi+2 \pi n) e^{-\frac{(\varphi+2 \pi n)^{2}}{4 \bar{s}}}}{\sqrt{\cos \sigma-\cos \varphi}}
$$


where now $\bar{s}=s l_{1}^{-2}$. Alternatively it can be represented in terms of the Legendre polynomials $P_{n}(\cos \sigma)$ as follows

$$
K_{S_{2}}\left(z, z^{\prime}, s\right)=\frac{1}{4 \pi l_{1}^{2}} \sum_{n=0}^{\infty}(2 n+1) P_{n}(\cos \sigma) e^{-\bar{s} n(n-1)} .
$$

The trace of the heat kernel on $2 \mathrm{~d}$ sphere then takes the form

$$
\begin{aligned}
& \operatorname{Tr} K_{S_{2}}(s)=\Theta_{S}\left(\bar{s} \frac{l^{2}}{l_{1}^{2}}\right), \\
& \Theta_{S}(s) \equiv \sum_{n=0}^{\infty}(2 n+1) e^{-s n(n+1)} .
\end{aligned}
$$

In eqs.(16) and (17), (18) $\sigma$ is the geodesic distance between the points $z$ and $z^{\prime}$ on $\mathrm{H}_{2}$ and $\mathrm{S}_{2}$ respectively. In particular, on $\mathrm{H}_{2}$ the geodesic distance $\sigma$ between the points $(x, \phi)$ and $(x, \phi+\Delta \phi)$ is given by

$$
\sinh ^{2} \frac{\sigma}{2}=\sinh ^{2} x \sin ^{2} \frac{\Delta \phi}{2}
$$

In principle, there must be a boundary at $x=x_{B}$ where some boundary conditions are imposed on the quantum scalar field. This would modify the heat kernel function (16). The boundary, however, can be arbitrarily far from the horizon at $x=0$. Since the entropy of the hole itself comes exclusively from the conical singularity at $x=0$ the boundary at $x=x_{B}$ will not to affect the entropy calculation. Therefore, we neglect the boundary and deal with the infinite space $\mathrm{H}_{2}$.

Hence the problem of evaluating the effective action (15) on $E^{\alpha}$ reduces to that of evaluating the heat kernel on the conical space $H_{2}^{\alpha}$. To find it we employ the Sommerfeld formula 13

$$
K_{H_{2}^{\alpha}}\left(z, z^{\prime}, s\right)=K_{H_{2}}\left(z, z^{\prime}, s\right)+\frac{\imath}{4 \pi \alpha} \int_{\Gamma} \cot \frac{w}{2 \alpha} K_{H_{2}}\left(\phi-\phi^{\prime}+w, s\right) d w,
$$

where $K_{H^{2}}$ is the heat kernel on regular manifold. The contour $\Gamma$ in (20) consists of two vertical lines, going from $(-\pi+\imath \infty)$ to $(-\pi-\imath \infty)$ and from $(\pi-\imath \infty)$ to $(\pi+$ $\imath \infty)$ and intersecting the real axis between the poles of the $\cot \frac{w}{2 \alpha}:-2 \pi \alpha, 0$ and $0,+$ $2 \pi \alpha$ respectively. For $\alpha=1$ the integrand in (20) is a $2 \pi$-periodic function and the contributions from these two vertical lines (at a fixed distance $2 \pi$ along the real axis) cancel each other. Applying the formula (20) we first define the quantity

$$
\operatorname{Tr}_{w} K_{H_{2}}=\int_{H_{2}} K_{H_{2}}\left(x=x^{\prime}, \phi^{\prime}=\phi+w\right) d \mu_{x}
$$


where $d \mu_{x}=l^{2} \sinh x d x d \phi$ is the measure on $H_{2}$. Inserting (16) into this expression, interchanging the $y$ and $x$ limits of integration, performing the $x$ and $\phi$ integrations, and then integrating by parts on $y$ yields

$$
\operatorname{Tr}_{w} K_{H_{2}}=\frac{e^{-\bar{s} / 4}}{(4 \pi \bar{s})^{1 / 2}} \int_{0}^{\infty} \frac{d y \cosh y e^{-y^{2} / \bar{s}}}{\left(\sinh ^{2} y+\sin ^{2} \frac{w}{2}\right)}
$$

Then the trace of the heat kernel on $H_{2}^{\alpha}$ can be evaluated as

$$
\operatorname{Tr} K_{H_{2}^{\alpha}}=\alpha \operatorname{Tr} K_{H_{2}}+\frac{\imath}{4 \pi} \int_{\Gamma} d w \cot \frac{w}{2 \alpha} \operatorname{Tr}_{w} K_{H_{2}}
$$

The contour integration is easily performed using the formula

$$
\frac{\imath}{4 \pi \alpha} \int_{\Gamma} \frac{\cot \frac{w}{2 \alpha} d w}{\sinh ^{2} y+\sin ^{2} \frac{w}{2}}=f(y, \alpha) \equiv \frac{1}{\sinh ^{2} y}\left(\frac{1}{\alpha} \frac{\tanh y}{\tanh \frac{y}{\alpha}}-1\right)
$$

obtained in [8]. Then we arrive at an integral representation for the heat kernel on $H_{2}^{\alpha}$ :

$$
\operatorname{Tr} K_{H_{2}^{\alpha}}=\alpha \operatorname{Tr} K_{H_{2}}+\alpha \frac{e^{-\bar{s} / 4}}{(4 \pi \bar{s})^{1 / 2}} \int_{0}^{\infty} d y \cosh y f(y, \alpha) e^{-y^{2} / \bar{s}}
$$

where $\bar{s}=s l^{-2}$. We are mainly interested in the second term in (23) which makes a contribution to the entropy. Using (23) and (19) we may find an integral representation for the effective action $W_{e f f}\left[E^{\alpha}\right](15)$. The quantum entropy $S_{q}$ can be calculated from $W_{\text {eff }}\left[E^{\alpha}\right]$ by making use the known formula

$$
S_{q}=\left.\left(\alpha \partial_{\alpha}-1\right) W_{e f f}\left[E^{\alpha}\right]\right|_{\alpha=1}
$$

Applying it to our case it is useful to note that the function (22) for $\alpha \simeq 1$ behaves as

$$
f(y, \alpha)=\frac{1}{\sinh ^{2} y}\left(1-\frac{2 y}{\sinh 2 y}\right)(1-\alpha)+O\left((1-\alpha)^{2}\right) .
$$

Then the quantum entropy is easily found to have the form

$$
S_{q}^{e x t}=\frac{1}{4 \sqrt{\pi}} \int_{\epsilon^{2} / l^{2}}^{\infty} \frac{d s}{s^{3 / 2}} k_{H}(s) \Theta_{S}\left(s \frac{l^{2}}{l_{1}^{2}}\right) e^{-s / 4}
$$

where

$$
k_{H}(s)=\int_{0}^{\infty} d y \frac{\cosh y}{\sinh ^{2} y}\left(1-\frac{2 y}{\sinh 2 y}\right) e^{-y^{2} / s} .
$$

The decrease of the integrand in (25) for large $s$ so rapid there is no need to introduce an infra-red cut-off to make the integral (25) convergent at large $s$. We see that the entropy 
$S_{q}$ (25) is a positive quantity which contains a divergent contribution in the limit $\epsilon \rightarrow 0$. This divergence can be isolated by using the asymptotic expansions

$$
k_{H}(s)=\sqrt{\pi s}\left(\frac{1}{3}-\frac{s}{20}+O\left(s^{2}\right)\right)
$$

and

$$
\Theta_{S}(s)=\frac{1}{s}\left(1+\frac{s}{3}+O\left(s^{2}\right)\right)
$$

yielding

$$
S_{\text {div }}^{\text {ext }}=\frac{1}{12} \frac{l_{1}^{2}}{\epsilon^{2}}+\left(\frac{1}{18}-\frac{1}{15} \frac{l_{1}^{2}}{l^{2}}\right) \ln \frac{l}{\epsilon}
$$

for the UV divergent contribution to (25).

Recall that in terms of the limiting metric (9) we have $l_{1}=r_{+}$and $l=b^{-1 / 2}$. For an extremal Reissner-Nordstrom black hole $l=l_{1}=r_{+}$and this expression becomes

$$
S_{d i v}^{e x t}=\frac{1}{12} \frac{r_{+}^{2}}{\epsilon^{2}}-\frac{1}{90} \ln \frac{r_{+}}{\epsilon}
$$

and coincides with the result (2) found earlier in [2].

According to general scheme described in ref. [14, the divergence (27) can be absorbed into the renormalization of the coupling constants in the gravitational effective action. After this procedure the total entropy $S=S_{B H}+S_{q}$ takes the form

$$
S^{e x t}\left(l, l_{1}\right)=\frac{A_{+}}{4 G}+\left(\frac{1}{18}-\frac{1}{15} \frac{l_{1}^{2}}{l^{2}}\right) \ln \frac{l}{\mu}+\Xi\left(\frac{l_{1}}{l}\right)
$$

where $\mu$ is a dimensional parameter, $A_{+}=4 \pi l_{1}^{2}$ is area of the horizon and the last term depends only on the ratio $\kappa=\frac{l_{1}^{2}}{l^{2}}$ :

$$
\Xi(\kappa)=\frac{1}{4 \sqrt{\pi}} \int_{0}^{\infty} \frac{d s}{s^{3 / 2}}\left(k_{H}(s) \Theta_{S}\left(\frac{s}{\kappa}\right)-\sqrt{\frac{\pi}{s}}\left(\left(\frac{1}{3}-\frac{s}{20}\right) \kappa+\frac{s}{9}\right)\right) e^{-s / 4} .
$$

The constant $G$ in (29) is the renormalized Newton constant. For simplicity we set to zero the renormalized values of the $R^{2}$-couplings in the gravitational effective action when derived (29). The expression (29) is the generic expression for the quantum corrected entropy of an extremal black hole. It may be understood as the universal limiting function of the entropy of a non-extremal black hole (3) in the extremal limit. In particular, when $l=l_{1}$ the last term (given by (30)) is an irrelevant constant and the entropy takes the form (1) modified by a logarithmic term

$$
S_{R N}^{e x t}=\frac{A_{+}}{4 G}-\frac{1}{180} \ln \frac{A_{+}}{\mu},
$$


where $A_{+}=4 \pi l^{2}$ is the horizon area.

A short digression is worth making here. There is a simpler way to get the expression (31). One should just notice that when geometry is characterized by just one dimensional parameter $l$ the latter can appear in the expression for the effective action $W_{\text {eff }}$ (13) and/or quantum entropy $S_{q}$ only in the ratio $\epsilon / l$ with the UV cut-off $\epsilon$. Thus all the dependence of the entropy or effective action on $l$ can be extracted by knowing only the divergent part of $S_{q}$ or $W_{\text {eff }}$ respectively. How it works for the extreme RN black hole is seen by comparing the eq.(2) and eqs. (28) and (31). This principle is universal and works not only for extreme holes but for any geometry characterized by just a single dimensional parameter. For extreme black holes one can exploit this to obtain the entropy without even knowing the limiting geometry. This observation is useful because (as we have already mentioned) not all the known black holes fall in the class described by the metric (3). In other cases the limiting procedure can be analyzed in the same way (see for example ref. [15]). However for the entropy calculation, we just need to know the UV divergence of the quantum entropy.

As an example consider the extreme dilaton black hole characterized by mass $m$ and electric charge $q$ where the extremality condition implies $2 m^{2}=q^{2}$. The horizon area vanishes in this case. Therefore the classical black hole entropy is zero. Nevertheless, the quantum entropy of the extreme dilaton hole does not vanish and has the UV divergent part calculated in [2] (equation (29) in [2]). The above arguments suggest that the total renormalized entropy of the extreme dilaton black hole is (up to an irrelevant constant)

$$
S_{d i l}^{e x t}=\frac{1}{18} \ln \frac{q}{\mu} .
$$

Another example is provided by the extreme Kerr-Newman (KN) hole characterized by zero electric charge $q$ and mass $m$ and rotation $a$ related as $m^{2}=a^{2}$. Then, using the eq.(4.12) of ref.[16] we find the total quantum entropy in this case to be

$$
S_{K N}^{e x t}=\frac{A_{+}}{4 G}+\frac{1}{90} \ln \frac{A_{+}}{\mu}
$$

where $A_{+}=4 \pi m^{2}$.

In a similar manner we can extract some information about entropy even in nonextremal cases. Using the expression for the divergence of the entropy of a $\mathrm{RN}$ hole 
(equation (20) in [2]) we find that the total quantum entropy in this case after UV renormalization takes the form

$$
S_{R N}=\frac{A_{+}}{4 G}+\left(\frac{2 r_{+}-3 r_{-}}{90 r_{+}}\right) \ln \frac{r_{+}}{\mu}+\Upsilon\left(\frac{r_{-}}{r_{+}}\right)
$$

where $r_{+}$and $r_{-}$are the outer and inner horizons. The function $\Upsilon\left(\frac{r_{-}}{r_{+}}\right)$can not be determined within this method.

Consider now how our results are modified to include the class of black holes with non-trivial horizon topology. The limiting geometry now is $E_{g}=H_{2} \times \Sigma_{g}$, where $\Sigma_{g}$ is a compact constant curvature surface of genus $g$. Calculating the effective action on $E_{g}^{\alpha}=H_{2}^{\alpha} \times \Sigma_{g}$ for arbitrary genus $g$ we need just to replace in (18) $\operatorname{Tr} K_{S_{2}}$ by the trace $\operatorname{Tr} K_{\Sigma_{g}}=\Theta_{\Sigma_{g}}$. For arbitrary $g$ the formula for the quantum entropy then becomes

$$
S_{q, g}^{e x t}=\frac{1}{4 \sqrt{\pi}} \int_{\epsilon^{2} / l^{2}}^{\infty} \frac{d s}{s^{3 / 2}} k_{H}(s) \Theta_{\Sigma_{g}}\left(s \frac{l^{2}}{l_{1}^{2}}\right) e^{-s / 4}
$$

generalizing the expression (25) for the spherical horizon topology. Remarkably, the heat kernel $K_{\Sigma_{g}}$ and its trace are known for arbitrary $g$.

The torus $T(g=1)$ with metric (10) is the plane $\mathcal{R}^{2}$ factored by an integral lattice $\Gamma, T \simeq \mathcal{R}^{2} / \Gamma$, that is, the points $\mathbf{z}$ and $\mathbf{z}+2 \pi \mathbf{n}$, with $2 \pi \mathbf{n} \in \Gamma$, are identified. The heat kernel on $T$ can be obtained from the heat kernel on $2 d$ plane by taking the infinite sum over images. The trace of the heat kernel then takes the form [12]

$$
\Theta_{T}(s)=\frac{A(T)}{4 \pi s} \sum_{2 \pi \mathbf{n} \in \Gamma} e^{-\frac{(2 \pi \mathbf{n})^{2}}{4 s}},
$$

where $A(T)$ is area of $T$. The function $\Theta_{T}(s)$ is actually a multidimensional Jacobi theta function. In the limit $s \rightarrow 0$ it behaves as

$$
\Theta_{T}(s)=\frac{A(T)}{4 \pi s}+O\left(e^{-\frac{1}{s}}\right)
$$

We may arrange the parameters of the lattice $\Gamma$ to have $A(T)=4 \pi$. The area of the horizon then is $A_{+}=4 \pi l_{1}^{2}$. The UV divergence of the quantum entropy (35) for the toroidal horizon topology is

$$
S_{\text {div }}^{g=1}=\frac{l_{1}^{2}}{12 \epsilon^{2}}-\frac{1}{15} \frac{l_{1}^{2}}{l^{2}} \ln \frac{l}{\epsilon}
$$


and the renormalized total entropy in the extremal limit reads

$$
S_{g=1}^{e x t}\left(l, l_{1}\right)=\frac{A_{+}}{4 G}-\frac{1}{15} \frac{l_{1}^{2}}{l^{2}} \ln \frac{l}{\mu}+\Xi_{g=1}\left(\frac{l_{1}}{l}\right)
$$

where the last term is function of only the ratio $\kappa=\frac{l_{1}^{2}}{l^{2}}$ :

$$
\Xi_{g=1}(\kappa)=\frac{1}{4 \sqrt{\pi}} \int_{0}^{\infty} \frac{d s}{s^{3 / 2}}\left(k_{H}(s) \Theta_{T}\left(\frac{s}{\kappa}\right)-\sqrt{\frac{\pi}{s}}\left(\left(\frac{1}{3}-\frac{s}{20}\right) \kappa\right)\right) e^{-s / 4} .
$$

The compact surface $\Sigma_{g}$ with genus $g \geq 2$ is identified with $H_{2} / \Gamma$, where $H_{2}$ is the hyperbolic space (usually represented as the upper half plane), $\Gamma$ is a discrete subgroup of $S L(2, \mathcal{R}) /\{ \pm 1\}$. The heat kernel on $\Sigma_{g}$ then is determined from the heat kernel on $H_{2}$ (16) by means the generalized procedure of summing over images. The trace of the heat kernel can be calculated using Selberg's trace formula [19], 20]:

$$
\Theta_{\Sigma_{g}}(s)=A\left(\Sigma_{g}\right) \frac{e^{-s / 4}}{(4 \pi s)^{3 / 2}} \int_{0}^{\infty} \frac{d y y e^{-\frac{y^{2}}{4 s}}}{\sinh \frac{y}{2}}+\frac{1}{2} \sum_{n=1}^{\infty} \sum_{\gamma} \frac{l(\gamma)}{\operatorname{prim} \frac{n l(\gamma)}{2}} \frac{e^{-s / 4}}{(4 \pi s)^{3 / 2}} e^{-n^{2} l^{2}(\gamma) / s}
$$

Here the sum over $\gamma$ primitive indicates summing over all $\gamma \in \Gamma$ which are not powers of another element in $\Gamma$ with exponent $\geq 2$ (if $\gamma$ is primitive, $\gamma^{-1}$ is also counted as primitive), and for each $\gamma$ the corresponding length of a closed geodesic $l(\gamma)$ is given by $\cosh \frac{l}{2}=|\operatorname{tr} \gamma| / 2$. For area of $\Sigma_{g}$ we have $A\left(\Sigma_{g}\right)=2 \pi\left|\chi\left(\Sigma_{g}\right)\right|=4 \pi(g-1)$. Only the first term in (40) has a pole for small $s$ so that

$$
\Theta_{\Sigma_{g}}=\frac{(g-1)}{s}\left(1-\frac{s}{3}+O\left(s^{2}\right)\right)
$$

This helps us to find the UV divergence of the quantum entropy (35)

$$
S_{d i v}^{g \geq 2}=\frac{l_{1}^{2}}{12 \epsilon^{2}}-(g-1)\left(\frac{1}{18}+\frac{1}{15} \frac{l_{1}^{2}}{l^{2}}\right) \ln \frac{l}{\epsilon} .
$$

After renormalization the total entropy of a black hole with higher genus horizon topology takes the following form in the extremal limit

$$
S_{g \geq 2}^{e x t}\left(l, l_{1}\right)=\frac{A_{+}}{4 G}-(g-1)\left(\frac{1}{18}+\frac{1}{15} \frac{l_{1}^{2}}{l^{2}}\right) \ln \frac{l}{\mu}+\Xi_{g \geq 2}\left(\frac{l_{1}}{l}\right),
$$

where

$$
\Xi_{g \geq 2}(\kappa)=\frac{1}{4 \sqrt{\pi}} \int_{0}^{\infty} \frac{d s}{s^{3 / 2}}\left(k_{H}(s) \Theta_{\Sigma_{g}}\left(\frac{s}{\kappa}\right)-(g-1) \sqrt{\frac{\pi}{s}}\left(\left(\frac{1}{3}-\frac{s}{20}\right) \kappa-\frac{s}{9}\right)\right) e^{-s / 4}
$$


The expression (25) and its generalization (35) are our main results. They give the universal behavior of the quantum entropy of a black hole in the extremal limit. The UV divergences of the entropy (27), (37) and (40) are in agreement with the general formula given in [2]. The equations (25), (35) can be generalized to the metric (8), (12) for arbitrary values of the parameter $c$. This, however, involves problem of the calculating the heat kernel on the $2 \mathrm{~d}$ sub-space $(x, \phi)$ which is not a space of constant curvature for $c \neq 1$. It is interesting to note that the entropy (25), (35) is completely determined by the spectral geometry of the horizon surface $\Sigma_{g}$ that is encoded in the theta function $\Theta_{\Sigma_{g}}(s)$. In principle, we could repeat our calculation for a black hole whose horizon is an arbitrary not necessarily symmetric $2 \mathrm{~d}$ surface of genus $g$. Our general expression (35) for the entropy is valid for this case as well. However, the calculation of the function $\Theta_{\Sigma_{g}}(s)$ becomes considerably more complicated. We may conclude that in the extreme limit the quantum entropy of a black hole is effectively "two-dimensional" and is determined by the geometry of the horizon. This property of the entropy becomes even more apparent in the case of so-called ultra-extreme black holes for which $b=0$ in (雨) in the extreme state [21]. The extreme geometry [5]

$$
d s^{2}=x^{2} d \phi^{2}+d x^{2}+r_{+}^{2} d \omega_{(g)}^{2}
$$

then describes the direct product space $U=\mathcal{R}^{2} \times \Sigma_{g}$ of 2 d plane $\mathcal{R}^{2}$ and compact surface $\Sigma_{g}$. The whole quantum analysis we have done before is simplified for the metric (44). This is because the heat kernel on the conical plane $\mathcal{R}_{\alpha}^{2}$ has the simple form

$$
\operatorname{Tr} K_{\mathcal{R}_{\alpha}^{2}}=\alpha \operatorname{Tr} K_{\mathcal{R}^{2}}+d(\alpha), d(\alpha)=\frac{1-\alpha^{2}}{12 \alpha}
$$

It can be obtained by taking accurately the limit $l \rightarrow \infty$ in the equation (23). Using this expression we find for the effective action on $U^{\alpha}$

$$
W_{e f f}\left[U^{\alpha}\right]=\left.\frac{1}{2} \ln \operatorname{det}(-\square)\right|_{U^{\alpha}}=\left.\alpha \ln \operatorname{det}(-\square)\right|_{U}+\left.d(\alpha) \ln \operatorname{det}(-\square)\right|_{\Sigma_{g}}
$$

where the last term is the logarithm of determinant of the Laplace operator $-\square$ defined on $\Sigma_{g}$. The quantum entropy then takes an especially simple form

$$
S_{q}^{u e x t}=\frac{1}{12} \int_{\frac{\epsilon^{2}}{r_{+}^{2}}}^{\infty} \frac{d s}{s} \Theta_{\Sigma_{g}}(s)
$$


and is expressed as the determinant of the two-dimensional Laplace operator

$$
S_{q}^{u e x t}=-\left.\frac{1}{6} \ln \operatorname{det}(-\square)\right|_{\Sigma_{g}}
$$

considered on the horizon surface $\Sigma_{g}$. So, as we can see from (48), the quantum entropy is purely "two-dimensional" in the ultra-extreme case.

To summarize, we considered the extreme limit of a black hole geometry of the RN type. Universally, the limiting geometry is the direct product of two 2-dimensional spaces and is characterized by just a few parameters corresponding to their length scales and the amount by which one of them is not a space of constant negative curvature (the other 2space has been assumed to have constant curvature). The quantum entropy of a massless scalar field possesses a corresponding universality in the extremal limit. The form of the limiting entropy is found (25) as function of the parameters of the limiting geometry. We have generalized it to black holes whose horizons have toroidal or higher genus topology (35). In general, the extreme quantum entropy is completely determined by the spectral geometry of the horizon. In the ultra-extreme case it is just the determinant of the twodimensional Laplacian. As a byproduct of our consideration we obtained expressions for the quantum entropy of black holes which are not of the RN type: extreme dilaton and extreme KN holes. In both cases the classical BH entropy is modified by some logarithmic corrections. Our results do not apply directly to extreme black holes in superstring theory [4] though an appropriate extension to this case might be an interesting problem.

\section{Note added:}

After this paper was complete we became aware of the recent preprint [22] where the quantum entropy is found to vanish in the extremal limit. We comment here on why this apparent disagreement is not in contradiction with our results.

In ref. [22] the quantum thermodynamical entropy is calculated by making use of the equation (eq.(10) in [22]) $\partial_{r_{+}} S_{q}=\eta$ where $\eta$ is constructed from the renormalized stress-energy tensor. Integrating this equation

$$
S_{q}=\int^{r_{+}} \eta+C
$$


produces an arbitrary constant $C$. This is a reasonable ambiguity since the thermodynamical entropy is always defined up to an irrelevant constant. However in ref. [22] this ambiguity is used to choose $C=C\left(r_{B}\right)$ so that for a generic non-extreme hole the entropy (eq.(11) in 22]) is defined to be

$$
S_{q}=\int_{r_{B}}^{r_{+}} \eta
$$

which vanishes when the size of the system $\left(r_{B}-r_{+}\right)$tends to zero.

The system under consideration represents a rather complicated interaction of two objects: a black hole and a hot gas (for a discussion of this see ref. [7]). Both objects contribute to the thermodynamical entropy $S_{q}$. The contribution of the hot gas can be identified and eliminated through its dependence on the size of the system. On the other hand, the contribution of the hole itself does not depend on this size. The method for calculating the entropy that we employ gives this contribution automatically. We believe that the vanishing of the entropy in the extremal case found in ref. 222] is a result of the above definition of $S_{q}$ that was used there. First of all, it contains both the black hole and hot gas contributions. Moreover, by adding the constant $C\left(r_{B}\right)$ (irrelevant for the entropy of a non-extreme hole) one effectively subtracts the contribution of the hole itself from the entropy of the extreme hole $\left(r_{B}=r_{+}\right)$in the definition of ref. [22]. By keeping $C$ an arbitrary constant and extracting the $r_{B}$-independent part in $S_{q}$ one would obtain the correct expression for the entropy of the hole itself that does not vanish, reproducing our result in the extreme limit.

\section{Acknowledgements}

This work was supported by the Natural Sciences and Engineering Research Council of Canada and by a NATO Science Fellowship.

\section{References}

[1] J. D. Bekenstein, Lett. Nuovo Cim. 4, 737 (1972); Phys. Rev. D7, 2333 (1973); S. W. Hawking, Comm. Math. Phys. 43, 199 (1975).

[2] S. N. Solodukhin, Phys. Rev. D51, 618 (1995). 
[3] S. W. Hawking, G. T. Horowitz and S. F. Ross, Phys. Rev. D51, 4302 (1995), G. W. Gibbons and R. E. Kallosh, Phys. Rev. D51, 2839 (1995); C. Teitelboim, Phys. Rev. D51, 4315 (1995).

[4] A. Strominger and C. Vafa, Phys. Lett. B379, 99 (1996);

[5] O. B. Zaslavskii, gr-qc/9707015, to appear in Phys. Rev. D.

[6] T. A. Jacobson, G. Kang and R. C. Myers, Phys. Rev. D49, 6587 (1994); D. V. Fursaev and S. N. Solodukhin, Phys. Rev. D52, 2133 (1995).

[7] V. P. Frolov, W. Israel and S. N. Solodukhin, Phys. Rev. D54, 2732 (1996).

[8] R. B. Mann and S. N. Solodukhin, Phys. Rev. D55, 3622 (1997).

[9] J.-G. Demers, R. Lafrance and R.C. Myers, Phys. Rev. D52, 2245 (1995).

[10] R. Kallosh, Phys. Lett. B282, 80 (1992).

[11] R. B. Mann, Class. Quant. Grav. 14 (1997) L109; D. R. Brill, J. Louko and P. Peldan, gr-qc/9705012, gr-qc/9705007; L. Vanzo, gr-qc/9705004.

[12] R. Camporesi, Phys. Rep. 196, 1 (1990).

[13] A. Sommerfeld, Proc. Lond. Math. Soc. 28, 417 (1897); H. S. Carslaw, Proc. Lond. Math. Soc. 20, 121 (1898); 8, 365 (1910); 18, 291 (1919); J. S. Dowker, J. Phys. A10, 115 (1977).

[14] D. V. Fursaev and S. N. Solodukhin, Phys. Lett. B365, 51 (1996),

[15] O. B. Zaslavskii, gr-qc/9707019.

[16] R. B. Mann and S. N. Solodukhin, Phys. Rev. D54, 3932 (1996).

[17] D. V. Fursaev, Mod. Phys. Lett. A10, 649 (1995).

[18] G. Cognola, L. Vanzo and S. Zerbini, Phys. Rev. D52, 4548 (1995).

[19] H. P. McKean, Commun. Pure and Appl. Math. 25, 225 (1972). 
[20] E. D'Hoker and D. H. Phong, Comm. Math. Phys. 104, 537 (1986).

[21] D. R. Brill and S. A. Hayward, Class. Quant. Grav. 11, 359 (1994).

[22] O. B. Zaslavskii, gr-qc/9708027. 